\title{
EFEITO DA COBERTURA VIVA COM LEGUMINOSAS HERBÁCEAS PERENES NA AGREGAÇÃO DE UM ARGISSOLO(1)
}

\author{
A. PERIN (2), J . G. M. GUERRA(3), M. G. TEIXEIRA(3), \\ M. G. PEREIRA(4) \& A. FONTANA(5)
}

\begin{abstract}
RESUMO
O presente trabalho foi desenvolvido na área do Campo Experimental da E mbrapa Agrobiologia, Seropédica (RJ ), com o objetivo de avaliar a morfologia e a distri bui ção de raízes de algumas legumi nosas herbáceas perenes; os efeitos da cobertura viva no teor de carbono orgânico, e a agregação de um Argissolo Vermel ho-Amarelo distrófico, medida pela estabilidade dos agregados em água. O delineamento experimental adotado foi o de blocos casualizados, com três repetições. Os tratamentos consistiram de três diferentes espécies de leguminosas herbáceas perenes e um tratamento-controle sem cobertura viva (capinado). As leguminosas utilizadas foram amendoim forrageiro (Arachis pintoi), cudzu tropical (Pueraria phaseoloides) e siratro (Macroptilium atropurpureum). Para a estabilidade de agregados, as profundidades de amostragem foram 0-5 e 5-10 cm, enquanto, para a morfologia e distribuição radicular, as avaliações consistiram das profundidades 0-5, 5-10, 10-20 e 20-40 cm. As coberturas com as leguminosas amendoim forrageiro e cudzu tropical propiciaram os maiores valores percentuais na classe de agregados $>2,00 \mathrm{~mm}$, em média 38 \% superiores aos obtidos na área capinada. Os valores do diâmetro médio ponderado (DMP) dos agregados no solo com cobertura de leguminosas foram superiores aos da área capi nada para ambas as camadas, o que demonstra o efeito favorável das coberturas vivas na estabilização dos agregados do solo. A cobertura viva de amendoim forrageiro proporcionou incremento no teor de carbono orgânico no solo. Quanto aos atri butos morfológicos das raízes, verificouse que $o$ amendoi $m$ forragei ro apresentou raio radicular intermediário entre as demais espécies eárea e massa radicular maiores, o que auxi liou na interpretação do efeito positivo da cobertura viva com essa espécie na agregação do solo.
\end{abstract}

Termos de indexação: adubação verde, matéria orgânica, estabilidade de agregados.

\footnotetext{
(1) Trabalho executado com recursos da Embrapa Agrobiologia, Seropédica (RJ ), subprojeto no 01.096.032-08. Recebido para publicação em julho de 2001 e aprovado em março de 2002.

(2) Doutorando em Fitotecnia, Departamento de Fitotecnia da Universidade Federal de Viçosa - UFV. CEP 36571-000 Viçosa (MG). E-mail: aperin@vicosa.ufv.br

(3) Pesquisador da Embrapa Agrobiologia. CEP 238900-000 Seropédica (RJ ). E-mail: gmguerra@cnpab.embrapa.br

(4) Professor Adjunto do Departamento de Sol os da UniversidadeFederal Rural do Rio deJ aneiro- UFRRJ . CEP 23890-000 Seropédica (RJ ). E-mail: gervasio@ufrrj.br

(5) Graduando de Agronomia, UFRRJ. Bolsista de Iniciação Científica, PIBIC/ CNPq. E-mail: fontana@ufrrj.br
} 


\title{
SUMMARY: EFFECT OF HERBACEOUS LEGUMINOUS SPECIES AS LIVE MULCHING ON SOIL AGGREGATION
}

\begin{abstract}
Tropical soils under continuous cultivation undergo fast degradati on, leading to crop production decline, mainly due to erosion and reduction of soil organic matter levels. Soil protection with livemulching is one of thealternatives to al leviatetheseeffects and to control soil degradation. This study was conducted to evaluatetheroot distribution and morphol ogy of three perennial herbaceous leguminous species, as well as its effects on soil aggregation and organic carbon content when used as livemul ching. Theexperiment was carried out on a Typic Haplustult soil in the experimental area of EMBRAPA Agrobiologia, Seropédica, State of Rio de J aneiro, Brazil, arranged in a completely randomized block design with three replicates. Treatments consisted of soil-covering with three herbaceous leguminous species (Arachis pintoi, Pueraria phaseol oides and Macroptilium atropurpureum) and a control treatment without livemulching, kept weed-free. Soil was sampled at depths of 0-5 and 5-10 cm for aggregatestabi lity analysis and at depths of 0-5; 5-10; $10-20$ and $20-40 \mathrm{~cm}$ for root distribution and morphology. A higher percentage of aggregates in the $>2.0 \mathrm{~mm}$ class was observed under A. pintoi and P. phaseoloides covers, $38 \%$ above the control treatment mean. The values of mean weigth diameter aggregates of soil for leguminous treatments were also higher than in the weeded area for both depths, demonstrating the favorable effect of live mulch on soil aggregate stability. An increase in the organic soil carbon level was observed for thetreatment with A. pintoi. Root morphol ogy analysis showed that A. pintoi presented an intermediateroot radius and higher root mass and area compared to other species, which may explain its positive effect on soil aggregation.
\end{abstract}

Index terms: green manure, organic matter, aggregatestability.

\section{NTRODUÇÃO}

O declínio da produtividade dos solos agrícolas de regiões tropicais e subtropicais cultivados continuamente tem sido atribuído princi palmenteà erosão e à redução dos níveis de matéria orgânica. A proteção do sol o com coberturas vivas ou mortas é uma das alternativas mais efetivas no controle de sua degradação. Neste sentido, sistemas de manejo que protejam o solo dos agentes climáticos e que proporcionem um contínuo aporte de resíduos orgânicos vêm sendo desenvolvidos e adaptados, dada a importância na formação de condições edáficas mais estáveis de produção. Castro Filho et al. (1998) reportam que, para um mesmo tipo de solo, diferentes práticas de manejo poderão afetar distintamente suas propriedades, incluindo os processos de agregação.

Do ponto de vista agrícola, a manutenção de uma boa estabilidade de agregados, conseqüentemente, a formação de boa estrutura, é condição primordial para garantir altas produtividades (Carpenedo \& Miel niczuk, 1990), haja vista queestas características estão relacionadas com a disponibilidade dear eágua às raízes das plantas, com osuprimento denutrientes, com a resistência mecânica do solo à penetração e com o desenvol vimento do sistema radicular (Baver et al., 1972; Tisdall \& Oades, 1979; Reid \& Goss, 1981).

Existem evidências de que as raízes aceleram a agregação, tanto pela liberação de exsudados orgânicos, como pela compressão das partículas e remoção de água, favorecendo a coesão entre as partículas do solo (Silva \& Mielniczuk, 1997; Silva et al., 1998; Castro Filho et al., 1998; Pal meira et al ., 1999). Na verdade, as raízes atuam na aproximação das partículas minerais pelas pressões exercidas no seu avanço através do espaço poroso do sol o, extração de água e liberação de substâncias orgânicas para a rizosfera (Miel niczuk, 1999). Essa ação simultânea de aproximação de partículas minerais e liberação de substâncias orgânicas no mesmo local, provavel mente, éa ação mais importante das raízes sobre a microagregação do solo (Haynes \& Francis, 1993).

Ao lado dessa atividade, que ocorre enquanto o sistema radicular está em crescimento, a matéria orgânica oriunda da decomposição do tecido radicular, raízes não-decompostas e mi céli os fúngicos também atuam na formação e estabilização, principalmente dos macroagregados. O material orgânico depositado continuamente serve como fonte de energia para os organismos do solo, cujos subprodutos, constituídos de moléculas orgânicas em diversas fases de decomposição, atuam comoagentes de estabilização de agregados pelas ligações de polímeros orgânicos com a superfície inorgânica por mei o de cáti ons pol ival entes (Tisdall \& Oades, 1982; Castro Filho et al., 1998).

O uso de leguminosas herbáceas perenes como cobertura viva permanente vem sendo avaliado como alternativa para proteção do solo em fruteiras. Esta 
prática tem caráter multifuncional, associando aspectos de conservação do solo e manutenção da fertilidade como conseqüência da adubação verde. Além de fixar $\mathrm{C}$ e $\mathrm{N}$ atmosféricos e maximizar a ciclagem denutrientes, favorecea atividadebiológica dosolo (Perin, 2001). Porém, o efeito das leguminosas perenes sobre características físicas, tais como: estabilização dos agregados do solo e distribuição de carbono nestas frações, é ainda pouco conhecido.

Conquanto na literatura sejam reportados inúmeros trabalhos sobre a caracterização do sistema radicular de plantas cultivadas (Cintra \& Miel niczuk, 1983), a disponibilidade de informações sobre características de raízes de leguminosas herbáceas perenes adaptadas a clima tropical é restrita (Andrade\& Valentim, 1999). Considerando que a prática de manutenção da cobertura viva permanente de solo em pomares pressupõe a consorciação da cobertura e frutífera, torna-se extremamenterelevante conhecer a distribuição das raízes destas leguminosas.

O presentetrabalho objetivou aval iar a morfologia e a distribuição de raízes de algumas leguminosas herbáceas perenes, os efeitos da cobertura viva no teor de carbono orgânico e na agregação de um Argissolo Vermel ho-A marel o distrófico.

\section{MATERIAL E MÉTODOS}

O trabalho faz parte de um experimento estabel ecido em fevereiro de 1995, na área do Campo Experimental da E mbrapa Agrobiol ogia, Seropédica (RJ ), em um Argissolo Vermel ho-Amarelo. Por ocasião da instalação do experimento, realizado em março de 1995, a área foi preparada de acordo com o sistema convencional, com uma aração e duas gradagens. A partir da instalação deste experimento de longa duração, não houve revolvimento do solo da área experimental, sendo o manejo das parcelas constituído pel o corteanual das plantas emanutenção dos resíduos vegetais na superfície do solo.

O delineamento experimental adotado foi o de blocos casualizados, com três repetições. Os tratamentos constaram de diferentes espécies de leguminosas herbáceas perenes e o controle sem cobertura viva (capinado). As leguminosas utilizadas foram amendoim forrageiro (Arachis pintoi ), cudzu tropical (Pueraria phaseoloides) e siratro (Macroptilium atropurpureum).

As amostras, coletadas em maio de 1999, foram tomadas nas camadas de 0-5 e 5-10 cm da superfície do sol o para avaliação do estado de agregação. Para cada amostra composta, foram coletadas quatro amostras simples, retirando-se os restos orgânicos da superfície. A determinação da estabilidade dos agregados em água foi feita segundo Kemper \& Chepil (1965), utilizando o aparelho de oscilação vertical (Yoder,1936). Para cada amostra de campo, foram feitas quatro repetições. Os valores obtidos foram usados para cálculo do diâmetro médio ponderado dos agregados (DMP), diâmetro médio geométrico dos agregados (DMG) e índice de estabilidade dos agregados (IEA) (Castro Filho et al., 1998). O DMP é tanto maior quanto maior for a percentagem de agregados grandes retidos nas peneiras com malhas maiores; o DMG representa uma estimativa da classe de agregados de maior ocorrência; o IEA representa uma medida de agregação total do sol o e não considera a distribuição por dasse deagregados. Quanto maior a quantidade de agregados $<0,105 \mathrm{~mm}$, menor será olEA. Essas propriedades foram calculadas por meio das seguintes equações:

$$
\mathrm{DMP}=\sum_{\mathrm{i}=1}^{\mathrm{n}}(\mathrm{xi} \text { wi })
$$

sendo

wi = propor ção de cada classe em relação ao total;

$\mathrm{xi}=$ diâmetro médio das classes $(\mathrm{mm})$

$$
\mathrm{DMP}=\operatorname{EXP} \sum_{\mathrm{i}=1}^{\mathrm{n}} \frac{\mathrm{wp} \log \mathrm{xi}}{\mathrm{wi}}
$$

em que

$$
\mathrm{wp}=\text { peso dos agregados de cada classe (g) }
$$

$$
\text { IEA }=\frac{(\text { Peso da amostra seca }- \text { wp } 105-\text { areia })}{\text { Peso da amostra seca }- \text { areia }} 100
$$

sendo

wp $105=$ peso dos agregados da classe $<105 \mathrm{~mm}$.

Para o cál culo do DMP, DMG eIEA, foi subtraída a percentagem de areia, partindo-se do princípio de que partícula unitária não é agregado (Palmeira et al., 1999).

O teor de carbono orgânico, em cada classe de agregados, foi determinado de acordo com EMBRAPA (1997).

Para avaliar a distribuição e morfologia do sistema radicular das leguminosas, for am col etadas amostras de solo em uma área com dimensões de $30 \times 30 \mathrm{~cm}$, nas profundidades de 0-5, 5-10, 10-20 e $20-40 \mathrm{~cm}$. E m seguida, as amostras foram cuidadosamente peneiradas, a fim de possibilitar a separação das raízes. Posteriormente, as raízes foram lavadas em água corrente e submetidas à determinação deárea radicular por mei ofotoelétrico, em aparelho Li-Cor 3100; volume radicular, com auxílio de proveta graduada; e massa radicular, seca em estufa de ventilação forçada de ar, mantida em temperatura de $65{ }^{\circ} \mathrm{C}$, durante $72 \mathrm{~h}$. A partir dos val ores de vol umee área radicular, estimou-se oraio radicular médio das raízes, por meio da equação $\mathrm{R}=((\mathrm{V} / \mathrm{A}) \times 2) \times 10$, sendo $\mathrm{R}$ : raio radicular médio $(\mathrm{mm})$; V: volume radicular $\left(\mathrm{cm}^{3}\right)$; A: área radicular $\left(\mathrm{cm}^{2}\right)$. 
Os procedimentos estatísticos foram realizados com auxílio do Sistema para Análises Estatísticas e Genéticas (SAEG), versão 5.0 (Euclydes, 1983), e constaram deanálisedevariância, aplicando-seoteste F para detectar significância nos níveis de $p<0,01$ e $p<0,05$. Constituíram fatores de análise os diferentes tratamentos, sendo as variáveis aval iadas separadamente em cada profundidade amostrada. Nas variáveis, em que o teste $F$ mostrou-se significativo, compararam-se as médias pelo teste deTukey $(p<0,05)$.

\section{RESULTADOS E DISCUSSÃO}

\section{Distribuição e morfologia radicular de leguminosas herbáceas perenes}

A avaliação de características morfológicas das raízes, distribuição espacial e massa radicular de leguminosas herbáceas perenes com potencial de utilização como cobertura viva permanente de solo em pomares é primordial para compreender os aspectos relacionados com a competição com a cultura principal. O conhecimento do sistema radicular podetambém subsi diar interpretações sob aspectos relacionados com compactação, agregação e comportamento térmico e hídrico do solo.

As raízes estimulam a agregação do solo, tanto pela promoção da população mi crobiana na rizosfera e pelo suprimento de resíduos orgânicos (Silva \& Mielniczuk, 1997), como pela promoçãoà reorientação eaproximação dos microagregados pela sua expansão e remoção de água, fazendo com que ocorra coesão entre as partículas do solo (Palmeira et al., 1999).

Quanto aos atributos morfológicos das raízes, verificou-se que o amendoim forrageiro, apesar de apresentar raio radicular intermediário entre as demais coberturas, revelou, em todas as profundidades, área e massa radicular superiores das demais leguminosas herbáceas perenes (Figuras 1 a 3). Estes valores encontrados no amendoim forrageiro foram $30 \%$ superiores aos obtidos por Andrade \& Valentim (1999). No entanto, vale destacar que o crescimento e a distribuição das raízes sofrem grande influência das propriedades físicas e químicas do solo (I to et al., 1996). Na camada de 0$5 \mathrm{~cm}$, a cobertura deamendoi m forrageiroapresentou massa radicular superior à do siratro $(23 \%)$ e do cudzu tropical (68\%). Em parte, as características do sistema radicular denso e bem desenvolvido do amendoim forrageiro explicam a maior agregação do solo, concordando com Stone \& Buttery (1989), os quais verificaram que espécies com maior massa radicular tenderam a aumentar a agregação. Segundo esses autores, a mel horia da agregação do solo pode ser um critério na sel eção de espécies a ser incluída no sistema de manejo, notadamenteem sistemas de recuperação de sol os degradados.

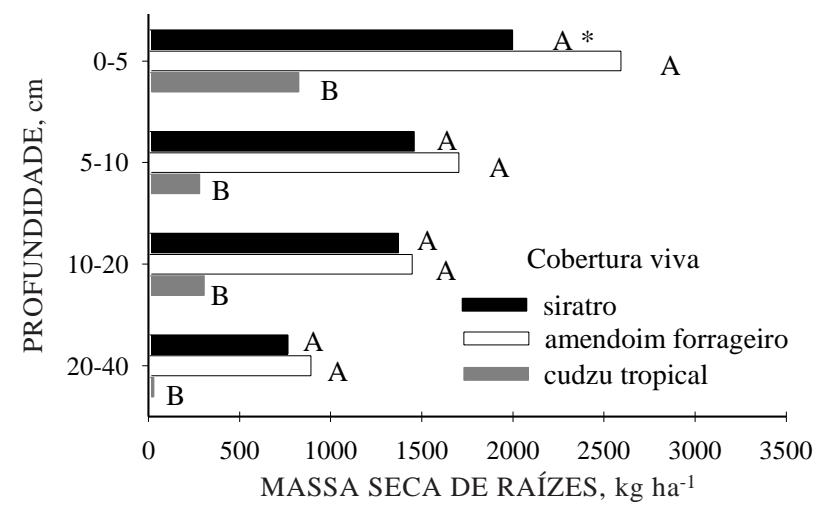

Figura 1. Distribuição de matéria seca de raízes de leguminosas herbáceas perenes usadas como cobertura viva permanente de solo. *Letras iguais na mesma profundidade de amostragem não diferem entre si pelo teste Tukey $(p<0,05)$.

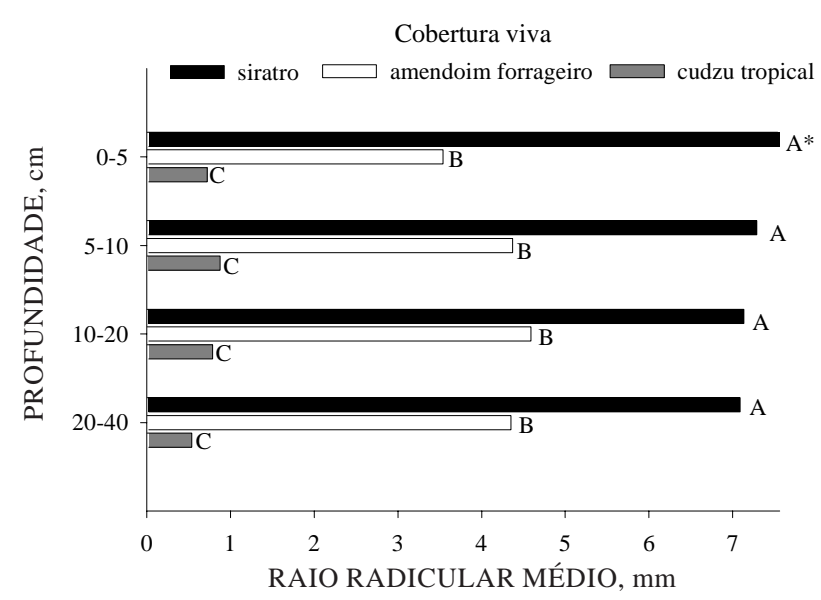

Figura 2. Raio médio de leguminosas herbáceas perenes usadas como cobertura viva permanente de solo. *Letras iguais na mesma profundidade de amostragem não diferem entre si pelo teste Tukey $(p<0,05)$.

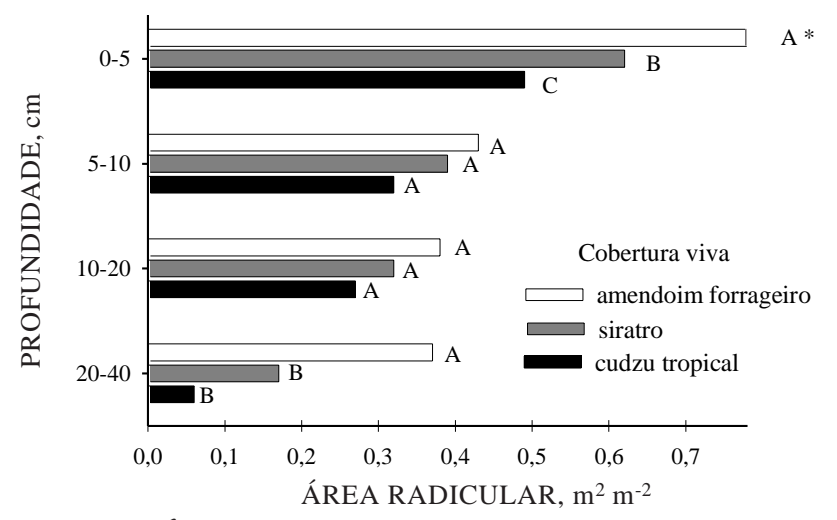

Figura 3. Área radicular de leguminosas herbáceas perenes usadas como cobertura viva permanente de solo. *Letras iguais na mesma profundidade de amostragem não diferem entre si pelo teste Tukey $(p<0,05)$. 
Resultados demonstraram, ainda, quea cobertura de amendoim forrageiro apresentou menor capacidade de manutenção de umidade do solo durante o período seco do ano, quando comparada à do siratro e cudzu tropical (Perin et al., 2000), e distribuição do sistema radicular com el evada capacidade de explorar o solo (Andrade \& Valentim, 1999), como forma de adaptação ao estresse hídrico. Estas características morfológicas conferidas pelo amendoim forrageiro evidenciam a mai or competição por água, quando consorciado com fruteiras com sistema radicular superficial.

Entretanto, quando a preocupação está voltada para a recuper ação de sol os com propriedades físicas degradadas, notadamentecom camada subsuperficial compactada e deficiente em $\mathrm{O}_{2}$, o uso do amendoim forrageiro pode ser mais eficiente que as demais leguminosas no rompimento destas camadas. Cintra \& Mielniczuk (1983), ao trabalharem com outros adubos verdes, identificaram a colza (Brassica napus) e o tremoço branco (L upinus albus) como as espécies com maior potencial na recuperação desol os compactados, considerando a maior produção de fitomassa subterrânea e a capacidade de tais espécies romper em camadas compactas.

\section{Distribuição dos agregados nas diferentes classes}

Tanto para a camada de $0-5 \mathrm{~cm}$ como a de $5-10 \mathrm{~cm}$ (Quadro 1), a maior proporção de agregados estáveis em água ocorreu na classe $>2 \mathrm{~mm}$ para ambos os tratamentos, concordando com resultados obtidos por Carpenedo \& Mielniczuk (1990), Paladini \& Mielniczuk (1991) e Silva \& Mielniczuk (1997). Ao comparar os val ores das áreas sob cobertura viva de leguminosas herbáceas perenes com os do tratamento-controle (capinado), verificou-se, para a camada de 0-5 cm, maior proporção de agregados para as leguminosas, notadamente na classe $>2,00 \mathrm{~mm}$ (Quadro 1), que foram, em média, $38 \%$ superiores aos da área capinada. J á para a camada de 5-10 cm, destacaram-se as coberturas formadas por amendoim forragei ro ecudzu tropical, sendo, em média, 33 \% superiores aos da área capinada (Quadro 1). Para as demais classes, com exceção da classe $<0,105 \mathrm{~mm}$ na camada de $5-10 \mathrm{~cm}$, foi constatado maior percentual de agregados na área capinada, indistintamente da camada avaliada, o que está associado possivel mente à menor estabilidade dos agregados para este tratamento.

Dentre as leguminosas, destaca-se a ação proporcionada pelo amendoim forrageiro e pelo cudzu tropical. O benefício proporcionado pelas leguminosas pode estar associado ao el evado potencial de produção de fitomassa (Guerra \& Teixeira, 1997; Espindola, 2001), presença de maior diversidade de organismos com características funcionais distintas (Merlim et al., 2000) e maior ocorrência de fungos micorrízicos arbusculares (Gravina, 1998), que promovem a estabilização dos agregados do sol o por meio da ação mecânica de seus micélios, enlaçando e unindo os microagregados (Bayer \& Mielniczuk, 1999). Pinotti \& Centurion (1999) detectaram maior estabilização dos agregados em sol o sob cobertura viva decudzu tropical associado

Quadro 1. Distribuição dos agregados nas diferentes peneiras nas camadas de 0-5 e de 5-10 cm da superfície do solo a partir de cobertura permanente de solo com leguminosas her báceas perenes

\begin{tabular}{|c|c|c|c|c|}
\hline \multirow{2}{*}{ Malha da peneira } & \multicolumn{4}{|c|}{ Cobertura viva de solo } \\
\hline & Amendoim forrageiro & Cudzu tropical & Siratro & Capinado \\
\hline \multirow[t]{2}{*}{$\mathrm{mm}$} & \multicolumn{4}{|c|}{$\%$} \\
\hline & \multicolumn{4}{|c|}{$0-5 \mathrm{~cm}$} \\
\hline$>2,00$ & $69,65 A^{(1)}$ & $71,17 \mathrm{~A}$ & $68,33 \mathrm{~A}$ & $50,40 \mathrm{~B}$ \\
\hline $1,00-2,00$ & $10,66 \mathrm{~B}$ & $8,43 \mathrm{~B}$ & $9,33 \mathrm{~B}$ & $11,47 \mathrm{~A}$ \\
\hline $0,50-1,00$ & $6,19 \mathrm{AB}$ & $7,03 \mathrm{~B}$ & $7,17 \mathrm{~B}$ & $12,03 \mathrm{~A}$ \\
\hline $0,25-0,50$ & $4,42 \mathrm{~B}$ & $4,93 \mathrm{~B}$ & $5,23 \mathrm{~B}$ & $8,43 \mathrm{~A}$ \\
\hline $0,105-0,25$ & $4,58 \mathrm{~B}$ & $5,03 \mathrm{~B}$ & $5,13 \mathrm{~B}$ & $7,10 \mathrm{~A}$ \\
\hline \multirow[t]{2}{*}{$<0,105$} & $4,50 \mathrm{~B}$ & $3,40 \mathrm{~B}$ & $4,80 \mathrm{~B}$ & $10,58 \mathrm{~A}$ \\
\hline & \multicolumn{4}{|c|}{$5-10 \mathrm{~cm}$} \\
\hline$>2,00$ & $66,05 A^{(1)}$ & $68,09 \mathrm{~A}$ & $58,27 \mathrm{~B}$ & $50,22 \mathrm{C}$ \\
\hline $1,00-2,00$ & $8,74 \mathrm{~B}$ & $6,93 \mathrm{~B}$ & $8,15 \mathrm{~B}$ & $14,27 \mathrm{~A}$ \\
\hline $0,50-1,00$ & $9,37 \mathrm{AB}$ & $7,03 \mathrm{~B}$ & $7,91 \mathrm{~B}$ & $12,03 \mathrm{~A}$ \\
\hline $0,25-0,50$ & $6,14 \mathrm{AB}$ & $5,31 \mathrm{~B}$ & $6,51 \mathrm{AB}$ & $8,53 \mathrm{~A}$ \\
\hline $0,105-0,25$ & $4,58 \mathrm{~B}$ & $6,26 \mathrm{AB}$ & $4,87 \mathrm{AB}$ & $7,22 \mathrm{~A}$ \\
\hline$<0,105$ & $5,12 \mathrm{~A}$ & $6,38 \mathrm{~A}$ & $14,30 \mathrm{~A}$ & $7,73 \mathrm{~A}$ \\
\hline
\end{tabular}

\footnotetext{
(1) Médias seguidas de letras iguais, na linha, não diferem entre si pelo teste Tukey $(p<0,05)$.
} 
ao cultivo de seringueira. Tais efeitos concordam com os resultados obtidos por Stone \& Buttery (1989), ao avaliarem a distri buição de agregados em sol o sob cobertura de nove espécies forrageiras.

\section{E feitos nos índices de agregação do solo}

Em relação ao diâmetro médio ponderado (DMP) dos agregados, verificou-se que o DMP foi estatisticamente superior nas áreas das leguminosas, quando estes valores foram comparados com os do tratamento-controle(Quadro 2). Deve-sedestacar que estes resultados decorrem do efeito proporcionado pelas leguminosas nas proporções de agregados mai ores e menores, respectivamente, para as classes de agregados $>2$ e $<0,105 \mathrm{~mm}$ (Quadro 1 ). Os efeitos destas coberturas vivas na agregação parecem ser devidos à ação concomitante das raízes e à deposição contínua de material orgânico na superfície do solo, mantendo um manto contra os efeitos indesejáveis do clima, além de estimular a atividade da fauna edáfica e de microrganismos organotróficos. E mbora nãotenham sido detectadas diferenças entre as leguminosas, verifica-se que o sol o sob cobertura de amendoim forrageiro tende a apresentar valores de DMP superiores aos das outras duas leguminosas. Gish \& Browning (1948), Horn $\&$ Dexter (1988) eH orn (1990) destacam que os cid os de umedecimento e secagem favorecem o processo de agregação do solo. Esses autores afirmam ainda que a desidratação de ligantes orgânicos e inorgânicos do solo proporcionam a formação de maior quantidade de agregados grandes.

Em relação às camadas amostradas, constatou-se, na camada de $0-5 \mathrm{~cm}$, quea presença das leguminosas influiu positivamente no DMP e no DMG; já para a camada de 5-10 cm, apenas oDMP foi favorecido pela presença das leguminosas (Quadro 2).

Estes resultados corroboram os de Silva \& Mielniczuk (1997), Silva et al. (1998), Castro Filho et al . (1998) ePal meira et al . (1999) que observaram o efeito das raízes na agregação.

\section{Distribuição do carbono orgânico nas classes de agregados}

Verificou-se, na camada de $0-5 \mathrm{~cm}$, queoamendoim forragei ro e o cudzu tropical, seguidos pel o siratro, proporcionaram os maiores valores de carbono orgânico, apresentando ambas as legumi nosas teores superiores aos da área capinada (Quadro 3). Na camada de 5-10 cm, as leguminosas também proporcionaram mai ores teores de carbono orgânico que da área capinada. Não ocorreu, entretanto, diferenças significativas entre as leguminosas. Comportamento semel hantefoi verificado por Duda (2000), em trabalho realizado na mesma área experimental em anos anteriores, observando quea cobertura de solo proporcionada pelas leguminosas amendoim forrageiro e siratro apresentaram maiores valores de carbono orgânico do que a área mantida capinada.

Resultados de pesquisa têm destacado que o amendoim forrageiro apresenta maior produção de fitomassa e estoque de nutrientes que outras leguminosas herbáceas perenes em condições edafoclimáticas semelhantes às realizadas neste trabal ho, bem como maior vel ocidade de decomposição dos resíduos de parte aérea (E spindola, 2001). Tais características podem contribuir no incremento de carbono orgânico ao solo, principal mente quando se maneja a cobertura com roçada e manutenção dos resíduos vegetais na área, o que potencializa o aporte de matéria orgânica na superfície o do solo.

Quanto à distribuição de carbono nas diferentes dasses de agregados, detectaram-se maiores valores na classe de agregados $>2,00 \mathrm{~mm}$, independentemente do tratamento ou da camada de sol o avaliada (Quadro 4). Este comportamento indica que o tamanho do agregado sofre influência do teor de

Quadro 2. Valores do diâmetro médio ponderado (DMP), diâmetro médio geométrico (DMG) e índice de estabilidade dos agregados (IEA) nas camadas de 0-5 e 5-10 cm da superfície do solo para as diferentes coberturas vivas de solo com leguminosas herbáceas perenes

Indice de estabilidade de agregados

\begin{tabular}{|c|c|c|c|c|c|c|}
\hline \multirow[t]{2}{*}{ Cobertura viva de solo } & \multicolumn{2}{|c|}{ DMP } & \multicolumn{2}{|c|}{ DMG } & \multicolumn{2}{|c|}{ IEA } \\
\hline & $0-5 \mathrm{~cm}$ & $5-10 \mathrm{~cm}$ & $0-5 \mathrm{~cm}$ & $5-10 \mathrm{~cm}$ & $0-5 \mathrm{~cm}$ & $5-10 \mathrm{~cm}$ \\
\hline & \multicolumn{4}{|c|}{$\mathrm{mm}$} & \multicolumn{2}{|c|}{$\%$} \\
\hline Amendoim forrageiro & $3,939 \mathrm{~A}^{(1)}$ & $3,522 \mathrm{~A}$ & $1,25 \mathrm{~A}$ & $1,08 \mathrm{~A}$ & $86,04 \mathrm{~A}$ & $84,40 \mathrm{~A}$ \\
\hline Cudzu tropical & $4,129 \mathrm{~A}$ & $3,472 \mathrm{~A}$ & $1,25 \mathrm{~A}$ & $1,10 \mathrm{~A}$ & $86,10 \mathrm{~A}$ & $85,48 \mathrm{~A}$ \\
\hline Siratro & $3,931 \mathrm{~A}$ & $3,133 \mathrm{AB}$ & $1,28 \mathrm{~A}$ & $1,10 \mathrm{~A}$ & $83,94 \mathrm{~A}$ & $83,14 \mathrm{~A}$ \\
\hline Capinado & $2,757 \mathrm{~B}$ & $2,708 \mathrm{~B}$ & $1,09 \mathrm{~B}$ & $1,08 \mathrm{~A}$ & $81,04 \mathrm{~A}$ & $80,80 \mathrm{~A}$ \\
\hline C.V. (\%) & 25,11 & 22,13 & 28,45 & 24,50 & 22,16 & 26,15 \\
\hline
\end{tabular}

(1) Médias seguidas de letras iguais, na coluna, não diferem pelo teste Tukey $(p<0,05)$. 
carbono orgânico, conforme destacado por Castro Filho et al. (1998). Constatou-se também que, na classe $>2,00 \mathrm{~mm}$, as leguminosas proporcionaram valores mais el evados de carbono que o sol o capinado, para ambas as camadas avaliadas. Para as demais classes de agregados, na camada de $0-5 \mathrm{~cm}$ e no intervalo de 0,25-0,50 mm, ocorreram diferenças significativas entre as coberturas de solo, apresentando as coberturas de cudzu tropical e siratro os menores valores $\left(4,89\right.$ a $\left.5,20 \mathrm{~g} \mathrm{~kg}^{-1}\right)$, enquanto, na camada de 5-10 cm, para este mesmo intervalo, apenas o siratro proporcionou os menores valores (Quadro 4).

Quadro 3. Teor de carbono orgânico $\left(\mathrm{g} \mathrm{kg}^{-1}\right)$ nos agregados das camadas de $0-5$ e $5-10 \mathrm{~cm}$ da superfície do sob os diferentes tipos de cobertura viva de leguminosas herbáceas perenes (média ponderada das classes de agregados)

\begin{tabular}{llc}
\hline & \multicolumn{2}{c}{ Carbono orgânico } \\
\cline { 2 - 3 } Cobertura viva de solo & $\mathbf{0 - 5} \mathbf{~ c m}$ & $\mathbf{5 - 1 0} \mathbf{~ c m}$ \\
\cline { 2 - 3 } & \multicolumn{2}{c}{$\mathbf{g ~ k g}^{-1}$} \\
\hline Amendoim forrageiro & $13,06 \mathrm{~A}^{(1)}$ & $12,63 \mathrm{~A}$ \\
Cudzu tropical & $12,77 \mathrm{AB}$ & $11,35 \mathrm{~A}$ \\
Siratro & $11,97 \mathrm{~B}$ & $11,83 \mathrm{~A}$ \\
Capinado & $9,62 \mathrm{C}$ & $9,28 \mathrm{~B}$ \\
C.V. (\%) & 16,89 & 15,92
\end{tabular}

(1) Médias seguidas de letras iguais, na coluna, não diferem entre si pelo teste Tukey $(p<0,05)$.

\section{CONCLUSÕES}

1. O solo sob cobertura viva de leguminosas herbáceas perenes apresentou maiores índices de agregação que a área capinada na camada de 0-5 cm.

2. As leguminosas herbáceas perenes, notadamente o amendoim forrageiro, contribuíram para o incremento de carbono orgânico do solo, tanto na camada de 0-5 como na de $5-10 \mathrm{~cm}$.

3. O amendoim forrageiro apresentou maior massa e área radicular do que siratro e cudzu tropical na camada de 0-40 cm da superfície do solo.

\section{LITERATURA CITADA}

ANDRADE, C.M.S. \& VALENTIM,J .F. Adaptação, produtividade e persistência de Arachis pintoi submetido à diferentes níveis de sombreamento. R. Bras. Zootec., 28:439-445, 1999.

BAVER, L.D.; GARDNER, W.H. \& GARDNER, W.R. Física de suelos. 4.ed. Mexico, Union Topografica Editorial Hipano Americana, 1972. 529p.

BAYER, C. \& MIELNICZUK, J. Dinâmica e função da matéria orgânica. In: SANTOS, G.A. \& CAMARGO, F.A.O., eds. Fundamentos da matéria orgânica do solo: ecossistemas tropicais e subtropicais. Porto Alegre, Genesis, 1999. p.926.

CINTRA, F.L. \& MIELNICZUK, J . Potencial de algumas espécies vegetais para a recuperação de solos com propriedades físicas degradadas. R. Bras. Ci. Solo, 7:197-201, 1983.

Quadro 4. Teor de carbono orgânico nas diferentes classes de agregados nas camadas de 0-5 e de 5-10 cm da superfície do solo em diferentes coberturas de solo com leguminosas herbáceas perenes

\begin{tabular}{|c|c|c|c|c|}
\hline \multirow{2}{*}{ Malha da peneira } & \multicolumn{4}{|c|}{ Carbono orgânico } \\
\hline & Amendoim forrageiro & Cudzu tropical & Siratro & Capinado \\
\hline \multirow[t]{2}{*}{$\mathrm{mm}$} & \multicolumn{4}{|c|}{$\mathrm{g} \mathrm{kg}^{-1}$} \\
\hline & \multicolumn{4}{|c|}{$0-5 \mathrm{~cm}$} \\
\hline$>2,00$ & $14,10 \mathrm{~A}^{(1)}$ & $13,43 \mathrm{~A}$ & $13,90 \mathrm{~A}$ & $11,33 \mathrm{~B}$ \\
\hline $1,00-2,00$ & $9,83 \mathrm{~A}$ & $8,43 \mathrm{~A}$ & $8,87 \mathrm{~A}$ & $9,57 \mathrm{~A}$ \\
\hline $0,50-1,00$ & $7,57 \mathrm{~A}$ & $6,70 \mathrm{~A}$ & $5,70 \mathrm{~B}$ & $7,00 \mathrm{AB}$ \\
\hline $0,25-0,50$ & $5,87 \mathrm{~A}$ & $5,30 \mathrm{~B}$ & $5,20 \mathrm{~B}$ & $6,80 \mathrm{~A}$ \\
\hline \multirow[t]{2}{*}{$0,105-0,25$} & $8,47 \mathrm{~A}$ & $6,60 \mathrm{~B}$ & $6,47 \mathrm{AB}$ & $7,93 \mathrm{~B}$ \\
\hline & \multicolumn{4}{|c|}{$5-10 \mathrm{~cm}$} \\
\hline$>2,00$ & $14,62 \mathrm{~A}^{(1)}$ & $14,01 \mathrm{~A}$ & $14,02 \mathrm{~A}$ & $10,99 \mathrm{~B}$ \\
\hline $1,00-2,00$ & $9,57 \mathrm{~A}$ & $7,75 \mathrm{~A}$ & $8,03 \mathrm{~A}$ & $9,57 \mathrm{~A}$ \\
\hline $0,50-1,00$ & $6,98 \mathrm{~A}$ & $5,46 \mathrm{AB}$ & $5,20 \mathrm{~B}$ & $6,47 \mathrm{AB}$ \\
\hline $0,25-0,50$ & $6,10 \mathrm{~A}$ & $5,10 \mathrm{AB}$ & $3,87 \mathrm{~B}$ & $5,97 \mathrm{~A}$ \\
\hline $0,105-0,25$ & $8,47 \mathrm{~A}$ & $6,60 \mathrm{~A}$ & $6,47 \mathrm{~A}$ & $7,31 \mathrm{~A}$ \\
\hline
\end{tabular}


CARPENEDO, V. \& MIELNICZUK, J. Estado de agregação e qualidade dos agregados de Latossolos Roxos, submetidos a diferentes sistemas de manejo. R. Bras. Ci. Solo, 14:99$105,1990$.

CASTRO FILHO, C.; MUZILLI, O. \& PODANOSCHI, A.L. Estabilidade dos agregados e sua relação com o teor de carbono orgânico em um Latossolo Roxo distrófico, em função de sistemas de plantio, rotações de culturas e métodos de preparo das amostras. R. Bras. Ci. Solo, 22:527538, 1998.

DUDA, G.P. Conteúdo de fósforo microbiano, orgânico e biodisponível em diferentes classes de solo. Seropédica, Universidade Federal Rural do Rio deJ aneiro, 2000. 158p. (Tese de Doutorado)

EMPRESA BRASILEIRA DE PESQUISA AGROPECUÁRIA EMBRAPA. Manual de métodos de análise química do solo. Rio de J aneiro, 1997. 212p.

ESPINDOLA, J.A.A. Avaliação de leguminosas herbáceas perenes usadas como cobertura viva de sol o e sua influência sobre a produção da bananeira (Musa spp.). Seropédica, Universidade Federal Rural do Rio deJ aneiro, 2001. 144p. (Tese de Doutorado)

EUCLYDES, R.F. Manual de utilização do programa SAEG (Sistema para análises estatísticas e genéricas). Viçosa, Universidade Federal de Viçosa, 1983. 59p.

GISH, E.H. \& BROWNING, G.M. Factors affecting the stability of soil agregates. Soil Sci. Soc. Am. Proc., 16:51-55, 1948.

GRAVINA, G.A. Avaliação da densidade de propágulos infectivos e capacidade infectiva de fungos micorrízicos arbusculares (FMA-s), em solos sob leguminosas herbáceas perenes. Seropédica, Universidade Federal Rural do Rio de J aneiro, 1998. 124p.

GUERRA, J.G.M. \& TEIXEIRA, M.G. Avaliação inicial dealgumas leguminosas herbáceas perenes para utilização como cobertura viva permanente de solo. Seropédica, EMBRAPA Agrobiologia, 1997. 7p. (Comunicado Técnico, 16)

HAYNES, R.J . \& FRANCIS, G.S. Changes in microbial biomass C, soil carbohydrate composition and agregate stability induced by growth of sel ected crop and forage species under field conditions. J. Soil. Sci., 44:665-675, 1993.

HORN, R. \& DEXTER, R.A. Dinamics of soil agregation in an irrigated desert loess. Soil Till. Res., 13:253-266, 1988.

HORN, R. Agregate caracterization as compared to soil bulk properties. Soil Till. Res., 17:265-289, 1990.

ITO, O.; KATAYAMA, K.; ADU-GYAMFI , J J .; DEVI, G. \& RAO, T.P. Root activities and function in component crops for intercropping. In: ITO, O.; J OHANSEN, C.;ADU-GYAMFI, J .J .; KATAYAMA, K.; RAO, J.V.D.K. \& REGO, T.J ., eds. Root and nitrogen in cropping systems of the semi-arid tropics. Tsukuba, J apan International Research Center for Agricultural Sciences, 1996. p.159-184.

KEMPER,W.D. \& CHEPIL, W.S. Size distribution of aggregates. In:BLACK, C.A.; EVANS, D.D.;WHITE, J.L.; ENSMINGER, L.E. \& CLARK, F.E., eds. Methods of soil analysis: physical and mineralogical properties, including statistics of measurement and sanpling. Part 1. Madison, American Society of Agronomy, 1965. p.499-510.
MERLIM, A.O.; AQUINO, A.M.; GUERRA, J.G.M. \& CORREA, M.E.F. Influência de diferentes coberturas vivas na diversidade da fauna do solo no cultivo de bananeira. In: REUNIÃO BRASILEIRA DE MANEJ O E CONSERVAÇÃO DE SOLO E ÁGUA, 13., Ilhéus, 2000. 500 anos de uso do solo. Resumos expandidos. Ilhéus, CEPLAC, 2000. (CDROM)

MIELNICZUK, J. I mportância do estudo de raízes no desenvolvimento de sistemas agrícolas sustentáveis. In: WORKSHOP SOBRE SISTEMA RADICULAR: METODOLOGIAS E ESTUDO DE CASO, Aracaju, 1999. Anais. Aracaju, EMBRAPA Tabulei ros Costeiros, 1999. p.1317.

PALADINI,F.C.S. \& MIELNICZUK, J. Distribuição de tamanho de agregados de um solo Podzól ico Vermel ho-Escuro afetado por sistemas de culturas. R. Bras. Ci. Solo, 15:135-140, 1991.

PALMEIRA, P.R.T.; PAULETTO, E.A.; TEIXEIRA, C.F.A.; GOMES, A.S. \& SILVA, J.B. Agregação de um Planossolo submetido a diferentes sistemas de cultivo. R. Bras. Ci. Solo, 23:189-195, 1999.

PERIN,A. Desempenho de leguminosas herbáceas perenes com potencial de utilização para cobertura viva e seus efeitos sobre alguns atributos físicos do solo. Seropédica, Universidade Federal Rural do Rio de J aneiro, 2001. 105p. (Tese de Mestrado)

PERIN, A.; GUERRA, J.G.M. \& TEIXEIRA, M.G. Efeito da morfologia radicular de leguminosas herbáceas perenes na umidade de um Argissolo. Seropédica, EMBRAPA Agrobiologia, 2000. 8p. (Comunicado Técnico, 44)

PINOTTI,A.A.R. \& CENTURION,J .F. Alterações na estabilidade de agregados do Latossolo Vermel ho-E scuro em função do manejo na entrelinha da seringueira. In: CONGRESSO BRASI LEIRO DE CIÊNCIA DO SOLO, 27., Brasília, 1999. Ciência do Solo e Qualidade de Vida: Resumos. Brasília, EMBRAPA Cerrados e Sociedade Brasileira de Ciência do Solo, 1999. (CD-ROOM)

REID, J.B. \& GOSS, M.J. Effect of living rots of different plant species on the aggregation stability of two arable soil. J. Soil Sci., 32:521-541, 1981.

SILVA, I.F. \& MIELNICZUK, J . Avaliação do estado de agregação do solo afetado pelo uso agrícola. R. Bras. Ci. Solo, 21:313319, 1997

SILVA, M.L.N.; BLANCANEAUX, P.; CURI, N.; LIMA, J.M.; MARQUES, J J.G.S.M. \& CARVALHO, A.M. Estabilidade e resistência de agregados de Latossolo Vermelho-Escuro cultivado com sucessão milho-adubo verde. Pesq. Agropec. Bras., 33:97-103, 1998.

STONE, J.A. \& BUTTERY, R. Nine forages and the agregation of a clay on soil. Can. J. Soil Sci, 69:165-169, 1989.

TISDALL,J .M. \& OADES,J .M. Organic matter and water- stable aggregates in soils. J. Soil Sci., 33:141-163, 1982.

TISDALL, J.M. \& OADES, J.M. Stabilization of soil aggregates by the root system of ryegrass. Aust. J. Soil Res., 17:429441, 1979.

YODER, R.E. A direct method of aggregate analysis of soil and a study of the physical nature of erosion losses. J. Am. Soc. Agron., 28:337-357, 1936. 